\title{
Influence of Ischemic and Infarcted Tissue on the Surface Potential
}

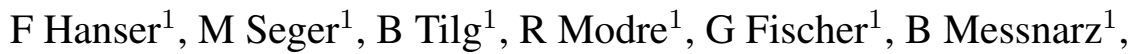 \\ F Hintringer $^{2}$, T Berger ${ }^{2}$, FX Roithinger ${ }^{2}$ \\ ${ }^{1}$ Institute for Medical Signal Processing and Imaging, \\ University for Health Informatics and Technology Tyrol, Innsbruck, Austria \\ ${ }^{2}$ Clinical Department of Cardiology, University Hospital Innsbruck, Austria
}

\begin{abstract}
The influence of ischemic and infarcted tissue on the body-surface potential was investigated. A cellular automaton was employed for modeling the heart's specialized conduction system, junction points, action potentials, and fiber orientation. The cellular automaton generated a transmembrane potential distribution providing the source distribution for the electrocardiographic forward problem. The body-surface potential was calculated with a bidomain-based forward model of a patient's volume conductor. Several scenarios were simulated including anterior and posterior myocardial infarction. The simulations demonstrated the influence on the body-surface potential displaying ST elevations and depressions as well as changes in polarity depending on the location of the ischemic region and electrode position.
\end{abstract}

\section{Introduction}

The modeling of ischemic and/or infarcted tissues and its influence on the body-surface ECG are of potential interest in many fields of cardiac electrophysiology. Electrocardiographic ST-segment changes during acute myocardial ischemia have been measured and studied by Horáček [1] in order to clarify the relationship of ST-segment elevations and depressions in various electrocardiographic leads. It was shown that a judicious choice of leads could improve and simplify the electrocardiographic criteria for recognizing acute myocardial infarction and for the infarct-related artery and the specific site of occlusion. The importance of anisotropy in modeling ST-segment shift was demonstrated by Johnston and Kilpatrick [2]. In addition, they also investigated the effect of conductivity values on ST segment shift in subendocardial ischemia [3]. In this work we present a model enabling the investigation of the influence of ischemic and/or infarcted tissue on the body-surface ECG depending on parameters like conductivity and anisotropy. The model comprises a heart model of the ventricle and a volume conductor model [4]. The heart model is based on a mesh generated with at least 25000 tetrahedron elements, and implements features like fiber architecture, specialized conduction system, conduction velocity, and refractory period. This cellular automaton generates a transmembrane potential distribution of the ventricle which provides the source distribution for the forward problem in order to calculate the body-surface potential.

\section{The model}

The model simulating the influence of ischemic and/or infarcted tissue on the body-surface potential comprises a model of the ventricle and the volume conductor. The former is used to generate a transmembrane potential distribution while the latter is needed to solve the electrocardiographic forward problem. Both models are described in the following two sections.

\subsection{Heart model}

The heart model was segmented from a patient's MRI short-axis scan and is based on a mesh with 25000 tetrahedron elements at least. The heart model considers electrophysiologically relevant structures including fiber architecture and specialized conduction system. The specialized conduction system considers the His bundle, left and right bundle branches, the Purkinje system, and junction points. Each structure can be modeled by assigning a certain material to each tetrahedron element. The number of materials is not limited. The presented heart model includes the following materials:

- Bundle of His

- Conjunction points

- Left bundle branch

- Right bundle branch

- Purkinje fiber

- Ventricular myocardium

- Ischaemic cells 
- Infarcted cells

Each material is characterized by the following properties and each property is described by one or more parameters:

- Material identification number

- Relationship between refractory period and heart rate (four parameters)

- Relationship between conduction velocity and heart rate (four parameters)

- Relationship between heart rate and action potential duration

- Action potential form (nine parameters)

- Conduction velocity parallel to fiber direction

- Conduction velocity normal to fiber direction

- Extracellular conductivity parallel to fiber orientation

- Extracellular conductivity normal to fiber orientation

- Intracellular conductivity parallel to fiber orientation

- Intracellular conductivity normal to fiber orientation

- Connections controlling conduction between materials

The ventricle's fiber orientation can be modeled interactively with a software package. One simply draws lines indicating the fiber orientation on the heart's surface as can be seen in Fig. 1. The local fiber orientation is then calculated by interpolating the user-input lines. We have been implementing some standard fiber orientation since the patient's actual fiber orientation is not easily accessible. Figure 2 shows a model of a patient's ventricle with vectors indicating the local fiber orientation. A detailed

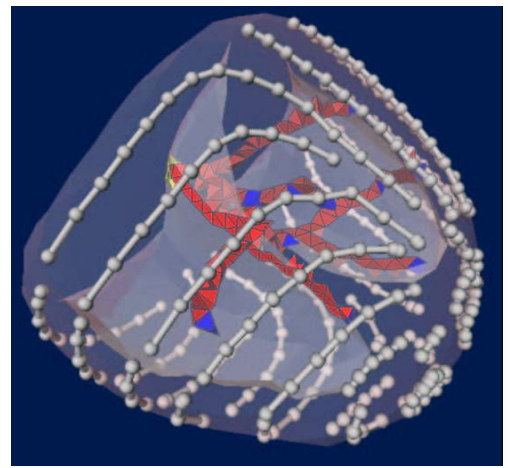

Figure 1. Lines indicating the user-defined fiber orientation on the ventricle's surface.

description of the interactive modeling of the ventricle's fiber orientation can be found in [5].

The ventricular depolarization and repolarization is calculated by a cellular automaton approach. A detailed description of the employed cellular automaton can be found in $[5,6,7,8]$. Figure 3 shows a ventricle depicting the His bundle, bundle branches, junction points, Purkinje fiber network, and ventricular myocardium.

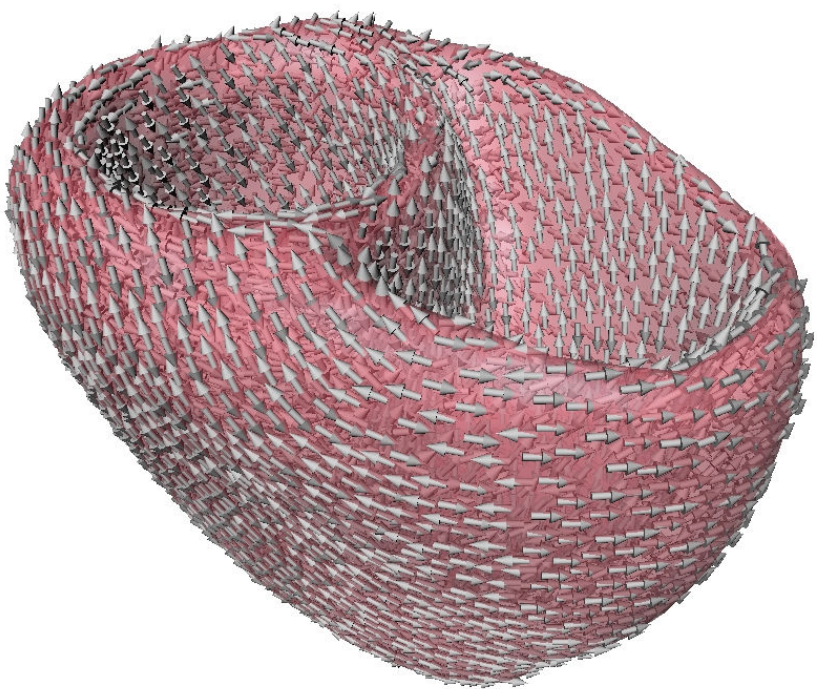

Figure 2. Fiber orientation of the employed ventricular heart model. Vectors indicate local fiber orientation.

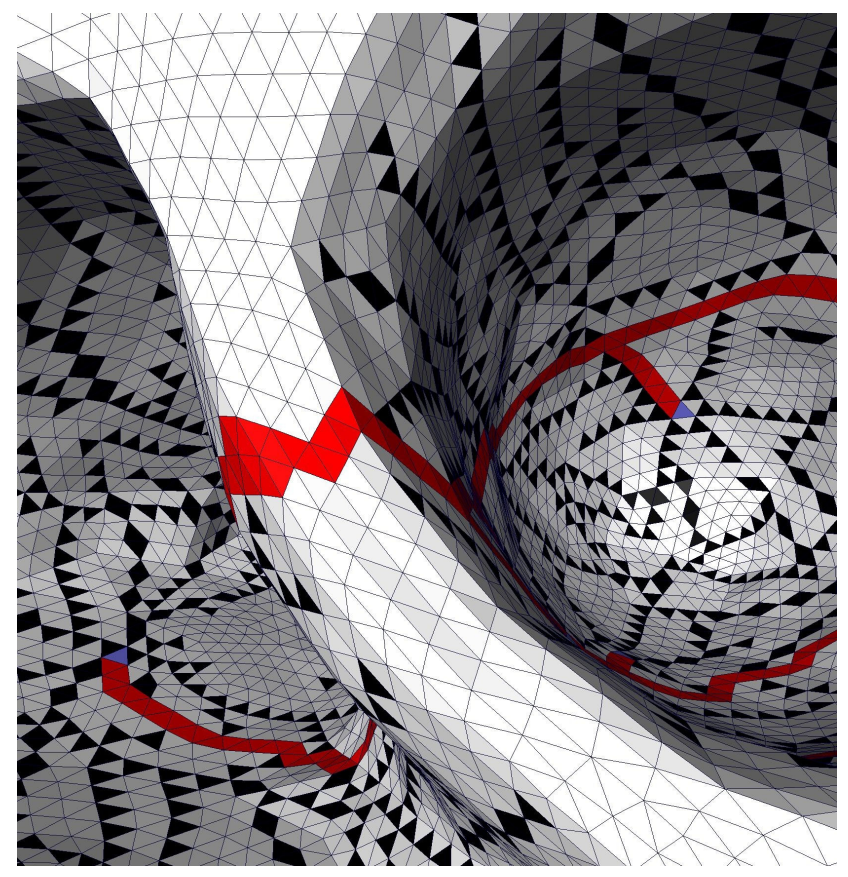

Figure 3. Ventricular heart model depicting His bundle, left and right bundle branches, junction points, Purkinje fiber network, and ventricular myocardium. Each structure is referred to as material with distinctive properties.

\subsection{Volume conductor}

The cellular automaton generates a transmembrane potential distribution of the ventricle which is used as input 
for the electrocardiographic forward problem in order to calculate the body-surface potential. Part of the forward problem is a patients's volume conductor model which considers the most electrodynamically relevant tissues or compartments. These compartments include the lungs, atrial and ventricular blood masses, active ventricular myocardium (same geometry as above), and the torso. Geometrical data were acquired with a 1.5Tesla MR scanner (Siemens Vision Plus). An axial scan of the torso and an additional short-axis scan of the heart (ECG-gated breath-hold oblique imaging mode) were used to extract the geometrical information. The entire volume conductor was modeled with more than a million tetrahedrons. As mentioned above, the different individual compartments comprised the torso, the lungs, and the blood masses. The associated conductivities were assumed to be 0.2 , 0.08 , and $0.6 \mathrm{Sm}^{-1}$, respectively. Conductivity values for the ventricle are listed below. Figure 4 shows a geometrical representation of a patient's volume conductor. The electrocardiographic forward problem is solved by a FEM approach.

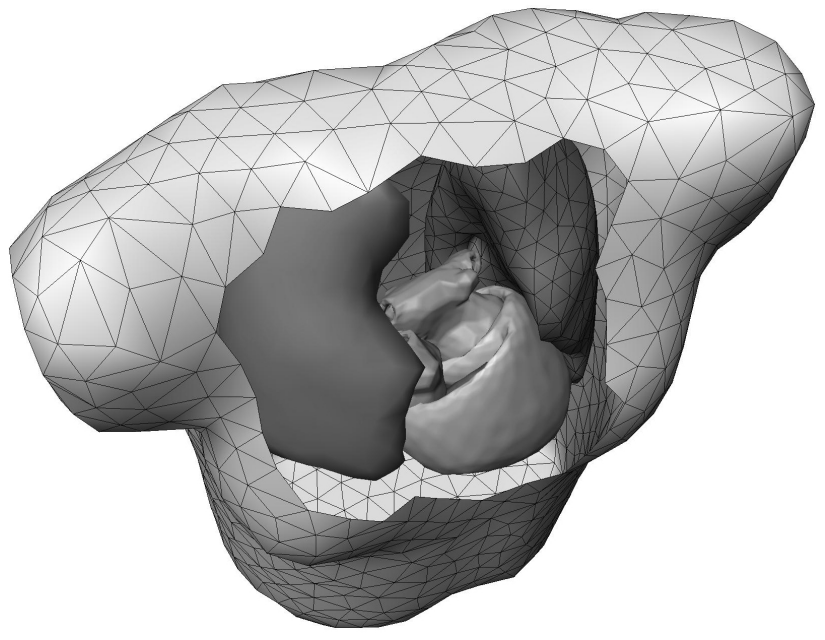

Figure 4. Geometrical visualization of an individual patient's volume conductor. The model depicts all electrodynamically relevant compartments: torso, ventricles, lungs, and blood masses. Only the compartment's surfaces are shown.

\section{Results}

We modeled ventricular myocardium with intracellular (in) and extracellular (ex) conductivities in a longitudinal (long) and transversal (trans) direction. For normal myocardium we used the following values (in $\mathrm{S} / \mathrm{m}$ ): 0.3 (in,long), 0.03 (in,trans), 0.6 (ex,long), and 0.15 (ex,trans). Ischemic tissue was modeled as follows: 0.175 (in,long), 0.04 (in,trans), 0.4 (ex,long), 0.175 (ex,trans). Infarcted tissue was modeled with 0.2 (ex), and 0 (in). We simulated some typical scenarios. We assumed, first, a myocardial infarction in the region of the left anterior descending coronary artery and, second, a posterior myocardial infarction. Figure 5 shows a geometrical representation of the ischemic and infarcted regions. The body-surface potential map of a sinus rhythm and those of the above mentioned posterior myocardial infarction are shown in Fig. 6. The body-surface ECG of one cardiac cycle shows Fig. 7. It shows ST-segment depression and morphological changes within the QRS complex. This was observed to be, however, strongly dependent on the position on the body surface which is in accordance with [1]. In another simulation we varied the resting potential of the ischemic cells. The associated body-surface potential is depicted in Fig. 8.
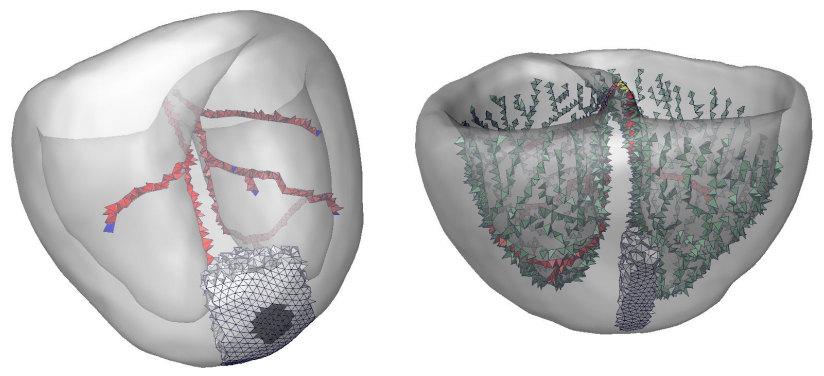

Figure 5. Left anterior myocardial infarction where we assumed both ischemic and infarcted tissue (left) and posterior myocardial infarction with ischemic tissue only.
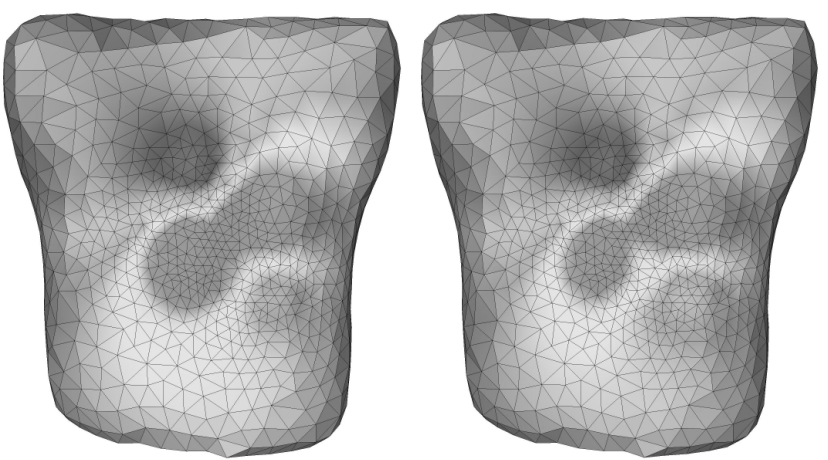

Figure 6. Body-surface potential map of a simulated sinus rhythm (left) and posterior myocardial infarction (right).

\section{Conclusion}

The presented heart model demonstrated its usefulness in studying the influence of ischemic and/or infarcted ventricular tissue on the body-surface ECG depending on various parameters like conductivity and resting potential. 


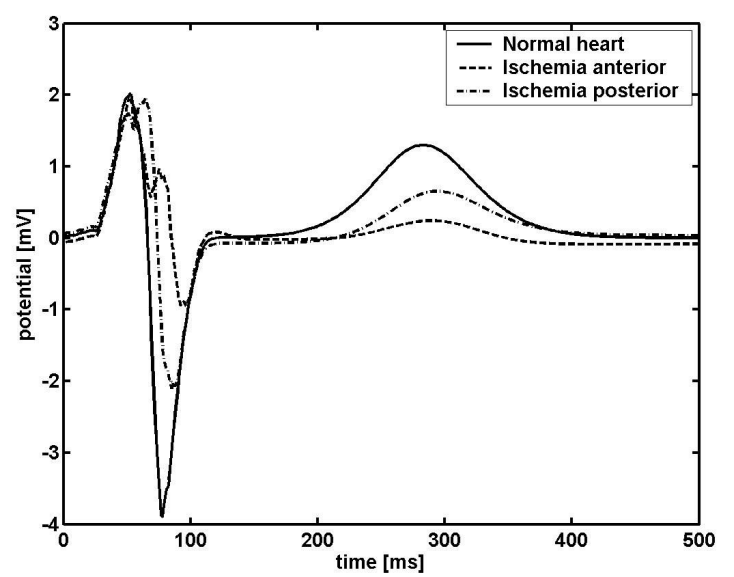

Figure 7. Body-surface potential of a site close to the precordial leads V2 and V3.

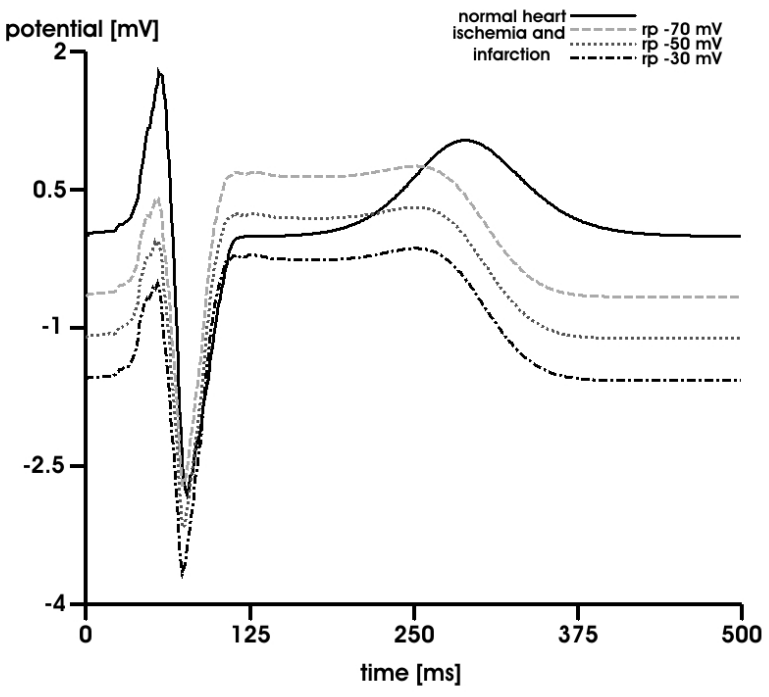

Figure 8. Simulated body-surface potentials of a site close to lead V1.

It was observed that ST-segment shifts, be it elevations or depressions, depend, in accordance to literature, on the location of the associated ischemic and/or infarcted region as well as on the location the body-surface potential is considered.

\section{Acknowledgements}

This work was supported by the START Y144-INF program funded by the Federal Ministry of Education, Science and Culture, Vienna, Austria.

\section{References}

[1] Horáček BM, Wagner GS. Electrocardiographic st-segment changes during acute myocardial ischemia. Cardiac Electrophysiology Review 2002;6:196-203.

[2] Johnston PR, Kilpatrick D, Li CY. The importance of anisotropy in modelling st segment shift in subendocardial ischaemia. IEEE Transaction on Biomedical Engineering December 2001;48(12):1366-1376.

[3] Johnston PR, Kilpatrick D. The effect of conductivity values on st segment shift in subendocardial ischaemia. IEEE Transaction on Biomedical Engineering 2003;50(2):150-158.

[4] Modre R, Tilg B, Fischer G, Wach P. An iterative algorithm for myocardial activation time imaging. Comput Methods Programs Biomed 2001;64:1-7.

[5] Hayn D. Ein Finite Elemente Herzmodell. Master's thesis, Graz Univertsity of Technology, March 2002.

[6] Killmann R. Numerisches Herzmodell. Master's thesis, Graz Univertsity of Technology, April 1987.

[7] Rosian M. Modellierung der Aktionspotentialformen im menschlichen Herzen. Master's thesis, Graz Univertsity of Technology, May 1991.

[8] Killman R. Three-Dimensional Numerical Simulation of the Excitation and Repolarisation Process in the Entire Human Heart with Special Emphasis on Reentrant Tachycardia. Ph.D. thesis, Graz Univertsity of Technology, October 1990.

Address for correspondence:

Friedrich Hanser, $\mathrm{PhD}$

Institute for Medical Signal Processing and Imaging University for Health Informatics and Technology Tyrol Innrain 98 / A-6020 Innsbruck/ Austria tel./fax: ++43-512-586734-817 / 850

friedrich.hanser@umit.at 